\title{
LAPTM4B Predicts Axillary Lymph Node Metastasis in Breast Cancer and Promotes Breast Cancer Cell Aggressiveness in Vitro
}

\author{
Min Xiao ${ }^{a}$ Shanshan Yang ${ }^{b}$ FanLing Meng ${ }^{b}$ Yu Qin ${ }^{c}$ Yue Yang ${ }^{d}$ Shusheng Jia ${ }^{a}$ \\ XiuMing Cai ${ }^{\mathrm{a}}$ Changli Lia Xiaoming Ning ${ }^{\mathrm{c}}$ Yuanxi Huanga \\ aDepartment of Breast Surgery, ${ }^{b}$ Department of Gynecology, 'Department of Pathology, The Affiliated \\ Tumor Hospital of Harbin Medical University, ${ }^{\mathrm{D} C a n c e r}$ Research Institute, Harbin Medical University, \\ Harbin, China
}

\section{Key Words}

Breast cancer • LAPTM4B • Axillary lymph node metastasis • EMT • Aggressiveness

\begin{abstract}
Purpose: Lysosome-associated protein transmembrane-4 beta (LAPTM4B) is associated with the prognosis of several human malignancies. In this study, the role of LAPTM4B in the metastatic potential of breast cancer $(B C)$ and its underlying molecular mechanisms were investigated. Methods: The relationship between LAPTM4B expression and axillary lymph node metastasis was determined in 291 BC specimens by immunohistochemistry. The expression of LAPTM4B in paired BC cells was overexpressed and inhibited to analyse the role of LAPTM4B in the aggressiveness of BC. Cell proliferation, migration and invasion were assessed in vitro. Metastasis-related protein levels were detected through Western blot. Results: Immunohistochemical staining demonstrated that high expression level of LAPTM4B was independently associated with axillary lymph node metastasis (odds ratio $=2.428 ; 95 \% \mathrm{CI}=1.333$ 4.425; $P=0.004)$. The LAPTM4B inhibition in MCF-7 cells inhibited cell proliferation, migration, invasion, and resulted in simultaneous downregulation of phosphorylated $\mathrm{N}$-cadherin, vimentin, and upregulation of E-cadherin. By contrast, the LAPTM4B overexpression promoted cell proliferation, migration, invasion, and led to simultaneous upregulation of $\mathrm{N}$-cadherin, vimentin, and downregulation of E-cadherin in T47D cells. Conclusions: High expression level of LAPTM4B predicts tumor metastatic potential in patients with BC. Our results provide the first evidence of the role of LAPTM4B as an Epithelial-mesenchymal transition (EMT) inducer that promotes aggressiveness in $\mathrm{BC}$ cells.

\section{Introduction}

Breast cancer $(\mathrm{BC})$ is the most frequently diagnosed malignancy in women worldwide and the second leading cause of cancer-related mortality among females in the United M. Xiao and S. Yang contributed equally to this manuscript. 
States [1]. Despite early detection and enhanced management, BC also accounted for $29 \%$ $(226,870)$ of the total new cases and $14 \%(39,510)$ of the total cancer deaths globally [2]. The presence of axillary lymph node metastasis is one of the most important factors in predicting the prognosis of patients diagnosed with BC. Therefore, the identification of potential markers for the early detection and prediction of axillary lymph node metastasis might play a significant role in predicting the prognosis and guiding the treatment of the breast cancer.

Epithelial-mesenchymal transition (EMT) is a critical event in developmental and tissue remodelling processes [3], EMT is also a crucial process for the invasion and migration of epithelial tumors [4]. Recent studies revealed that BC cells have undergone EMT to acquire aggressive malignant properties [5, 6]. As a novel tumor-associated gene, lysosomal protein transmembtane $4 \beta$ (LAPTM4B) was originally cloned in hepatocellular carcinomas by Rouli Zhou [7, 8]. LAPTM4B is mapped to chromosome 8q22.1, and composed of seven exons and six introns [8]. LAPTM4B is implicated in the tumorigenesis, promotion, metastasis and prognosis of many human malignancies [9-13]. In our previous study, we revealed that the expression level of LAPTM4B is high in patients with BC, and high level of LAPTM4B expression is closely associated with the progression and poor prognosis in patients with breast cancer [14]. However, up to date, there have been no published reports evaluating the its metastatic potential in $\mathrm{BC}$ cells, and the molecular mechanisms underlying its metastatic potential are poorly understood.

Herein, the present study aimed to assess the predicted significance of LAPTM4B in axillary lymph node metastasis in patients with $\mathrm{BC}$, and its potential oncogenic role in human $\mathrm{BC}$ cells, also we provided the evidence implicating the role of LAPTM4B in the metastatic potential of BC cells were via promoting the EMT.

\section{Materials and Methods}

\section{Patients and specimens}

291 Paraffin-embedded samples were obtained from patients with BC who were surgically treated in the Affiliated Tumor Hospital of Harbin Medical University between October 2005 and September 2007, including 194 breast cancer samples from our previous study about the association between the LAPTM4B expression and the prognosis of the breast cancer. None of the patients received chemotherapy, radiotherapy, or immunotherapy prior to surgery. All the patients with BC underwent primary mastectomies plus axillary lymph node dissection and the number of dissected lymph nodes was $>10$ [15]. And we detected the expression of LAPTM4B in 291 BC samples, 20 positive axillary lymph nodes, and 20 negative axillary lymph nodes.

This study complied with the Helsinki Declaration and was approved by the Institute Research Medical Ethics Committee of Harbin Medical University, China. Informed consent was obtained from all patients or their family members.

Immunohistochemical staining and evaluation

Formalin-fixed and paraffin-embedded samples were used for LAPTM4B immunohistochemical staining. After deparaffinage and blocking, the antigen-antibody reaction was incubated overnight at $4^{\circ} \mathrm{C}$. Diaminobenzidine (DAB) reagents were applied to detect the signal from the antigen-antibody reaction. All sections were counterstained with hema-toxylin. The primary anti-LAPTM4B rabbit monoclonal antibody (Bioss Inc., China) was used at a dilution of dilution 1:200. Primary antibody was replaced with the phosphate-buffered saline as a negative staining control, and the cervical cancer with positive LAPTM4B expression was considered as a positive control [11].

LAPTM4B staining was localized in the cytoplasm of positively stained immunoreactive cells. Staining intensity was graded on a 0-2 scale as follows: 0 (no staining or only weak staining=light yellow), 1 (moderate staining=yellow brown), and 2 (strong staining=brown). The percentage of positively stained immunoreactive cells was scored as follows: 0 ( $<10 \%$ positive tumor cells), 1 (10-50\% positive tumor cells), $2(>50 \%$ positive tumor cells). The staining score was calculated as the staining intensity score multiplied 


\section{Cellular Physiology Cell Physiol Biochem 2017;41:1072-1082 \begin{tabular}{ll|l} 
DOI: 10.1159/000464115 & 2017 The Author(s). Published by S. Karger AG, Basel \\
and Biochemistry Published online: February 27, 2017 & \begin{tabular}{l} 
www.karger.com/cpb \\
\cline { 2 - 3 }
\end{tabular}
\end{tabular} \\ Xiao et al.: LAPTM4B Promotes Breast Cancer Aggressiveness}

by the percentage score. This score ranged from 0 to 4 . Low expression was defined as a staining score of 0-2. High expression was defined as a staining score of 3-4. All sections were examined microscopically and scored by two independent pathologists who were blinded to the clinicopathological information and corresponding hematoxylin-eosin (H\&E) slides of the patients. In cases of significant disagreement, the scores in question were reviewed by the original two pathologists and a senior pathologist until a consensus was reached.

\section{Cell lines}

Three human BC cell lines (MCF-7, MDA-MB-231 and T47D cells) were obtained from the cell bank of the Committee on Type Culture Collection of the Chinese Academy of Sciences (Shanghai, China), and all cell lines were maintained in Dulbecco's Modified Eagle's Medium (Gibco, USA) Human BC ZR-75-30 cell line were obtained from Zhongqiaoxinzhou Biotechnology Co.Ltd. (Shanghai, China) and maintained in RPMI1640 medium (Gibco, USA). All media were supplemented with 10\% foetal bovine serum (FBS). All cell lines were cultured at $37^{\circ} \mathrm{C}$ in a humidified incubator with $5 \% \mathrm{CO}_{2}$.

\section{Cell culture and transfection studies}

We selected MCF-7 cells with high expression level of LAPTM4B for shRNA knockdown experiments after Western blot analysis. We also selected T47D cells with low expression level of LAPTM4B for upregulation experiments. The pSilencer-shRNA plasmids for knockdown of LAPTM4B were constructed with pSilencer ${ }^{\mathrm{TM}}$ hygro vectors (Ambion, Austin, TX) according to the manufacturer's instructions. The target sequence of LAPTM4B shRNA was ATGCTGTGGTACTGTTGATTT [16]. The complete open reading frame (ORF) of human LAPTM4B was polymerase chain reaction(PCR)-amplified from a cDNA clone from OriGene (NM_018407) using specific primers to introduce an EcoRI site at the 5'end and an XhoI site at the 3'end and cloned into the EcoRI and Sall sites of pEGFP-C2 vector (Clontech) [17]. Transient transfection was conducted with pEGFP-C2 vector, pEGFP-C2-LAPTM4B-AE or pSilencer, and pSilencer-LAPTM4B-shRNA. Over-expression and silencing efficiencies were determined by Western blot.

\section{Cell proliferation assay (MTT assay)}

The cell proliferation assay was performed using 3-(4,5-dimethylthiazol-2-yl)-2,5-diphenyltetrazolium bromide (MTT) assay (WLA021a, Wanleibio., Shenyang, China) according to the manufacturer's instructions. Briefly, the cells were plated in 96 -well plates at $3 \times 10^{3}$ cells per well. The cells were then cultured at $37^{\circ} \mathrm{C}$ in humidified incubator with $5 \% \mathrm{CO}_{2}$ for $24,48,72,96$, and $120 \mathrm{~h}$. On each day, cells were stained with $20 \mu \mathrm{l}$ MTT reagent $(5 \mathrm{mg} / \mathrm{ml})$ at $37^{\circ} \mathrm{C}$ for $4 \mathrm{~h}$ followed by removal of the culture medium and then resuspended in $150 \mu \mathrm{l}$ dimethyl sulfoxide for $10 \mathrm{~min}$. Spectrometric absorbance was measured at $490 \mathrm{~nm}$. Each experiment was repeated thrice.

\section{Wounding-healing assay}

Cell migration was assessed by measuring the movement of cells into a scraped wound. Cells were seeded at $3 \times 10^{5}$ cells/well in six-well tissue culture plates and starved overnight in the serum-free medium with $1 \mu \mathrm{g} / \mathrm{ml}$ mitomycin C. Subsequently, a cellular area was made by a $200 \mathrm{ml}$ pipette tube. The remaining cells were washed with PBS to remove cell debris and were then incubated at $37^{\circ} \mathrm{C}$ in a humidified atmosphere of $5 \% \mathrm{CO}_{2}$ in serum-free medium. Wound closure was observed after 24 and $48 \mathrm{~h}$. Images of the cells that had migrated into the cell-free scratch wound area were acquired and the migration distance was measured under inverted microscope. The scratch wound widths were determined by the relative percentage compared to untreated control cells. Each experiment was independently performed thrice.

\section{Transwell assay}

The invasive ability of cervical cancer cells was detected using 24-well transwell cell culture chambers (8.0 $\mu \mathrm{m}$ pore size, Costar, Cambridge, MA, USA) according to the manufacturer's instructions. The insert membranes were pre-coated with Matrigel (BD, San Diego, CA, USA). At $24 \mathrm{~h}$ post-transfection, the cells were seeded at an appropriate density $\left(1 \times 10^{5} / \mathrm{ml}\right)$ in the upper chamber and cultured in OPTI-MEM (Gibco, USA) without FBS for a further $24 \mathrm{~h}$. The cells were allowed to migrate toward the RPMI 1640 medium containing 20\% FBS in the bottom chamber. The non-migratory cells on the upper membrane surface were removed with a cotton tip. The migratory cells attached to the lower membrane surface were fixed with 


\section{Cellular Physiology Cell Physiol Biochem 2017;41:1072-1082 \begin{tabular}{ll|l} 
DOI: 10.1159/000464115 & $\begin{array}{l}\text { O } 2017 \text { The Author(s). Published by S. Karger AG, Basel } \\
\text { www.karger.com/cpb }\end{array}$ \\
\cline { 2 - 3 }
\end{tabular} \\ Xiao et al.: LAPTM4B Promotes Breast Cancer Aggressiveness}

4\% paraformaldehyde and stained with H\&E. The numbers of invaded cells were counted in five randomly selected fields under a microscope.

\section{Protein extraction and Western blot analysis}

Total protein was extracted and separated on $10 \%$ sodium dodecyl sulphate-polyacrylamide gel electrophoresis (SDS-PAGE) gels, transferred to polyvinylidene difluoride membrane (Millipore Company, Billerica, MA, USA), blocked with 5\% skimmed milk and incubated with primary antibodies targeting LAPTM4B, E-cadherin, N-catenin, and vimentin, in tris-buffered saline with tween (TBST) containing $1 \%$ bovine serum albumin (BSA) overnight at $4^{\circ} \mathrm{C}$ followed by incubation with the horseradish peroxidaselabeled secondary antibody in that order. The specific antibody to $\beta$-actin (WL0001, Wanlei Bio, Shenyang, China) was used as gel loading control.

\section{Statistical analysis}

The association between the LAPTM4B protein and axillary lymph node metastasis was assessed by univariate and multivariate logistic regressions with covariate adjustment. Quantitative data are expressed as mean $\pm \mathrm{SD}$. The Student's t-test was used to compare quantitative data populations with normal distributions and equal variances. Statistical analysis was performed using SPSS Version 16.0 for Windows. All tests were two-tailed. $P<0.05$ was considered statistically significant.

\section{Results}

Effect of high LAPTM4B expression level on axillary lymph node metastasis in BC

Similar to our previous study, the LAPTM4B staining was localized within the cytoplasm of immunoreactive cells (Fig. 1), the high LAPTM4B expression level was correlated with large tumor size $(P=0.003)$, advanced TNM stage $(P<0.001)$, axillary lymph node metastasis $(P<0.001)$, and postoperative recurrence $(P=0.007)$, as shown in Table 1 . Of the 168 patients with axillary lymph node metastasis, 140 (83.3\%) patients exhibited high LAPTM4B expression level, and of the 123 patients without axillary lymph node metastasis, 44(35.8\%) patients had low LAPTM4B expression. The sensitivity and specificity of high LAPTM4B ex-

Fig. 1. Representative immunostaining for LAPTM4B expression in breast cancer. $(\mathrm{A}, \mathrm{a}$ and $\mathrm{B}$, b) high LAPTM4B expression in breast cancer; (C, c) high LAPTM4B expression in metastatic axillary lymph node of breast cancer (D, d and E, e) low expression of LAPTM4B in breast cancer; (F, f) low LAPTM4B expression in nomal axillary lymph node of breast cancer.

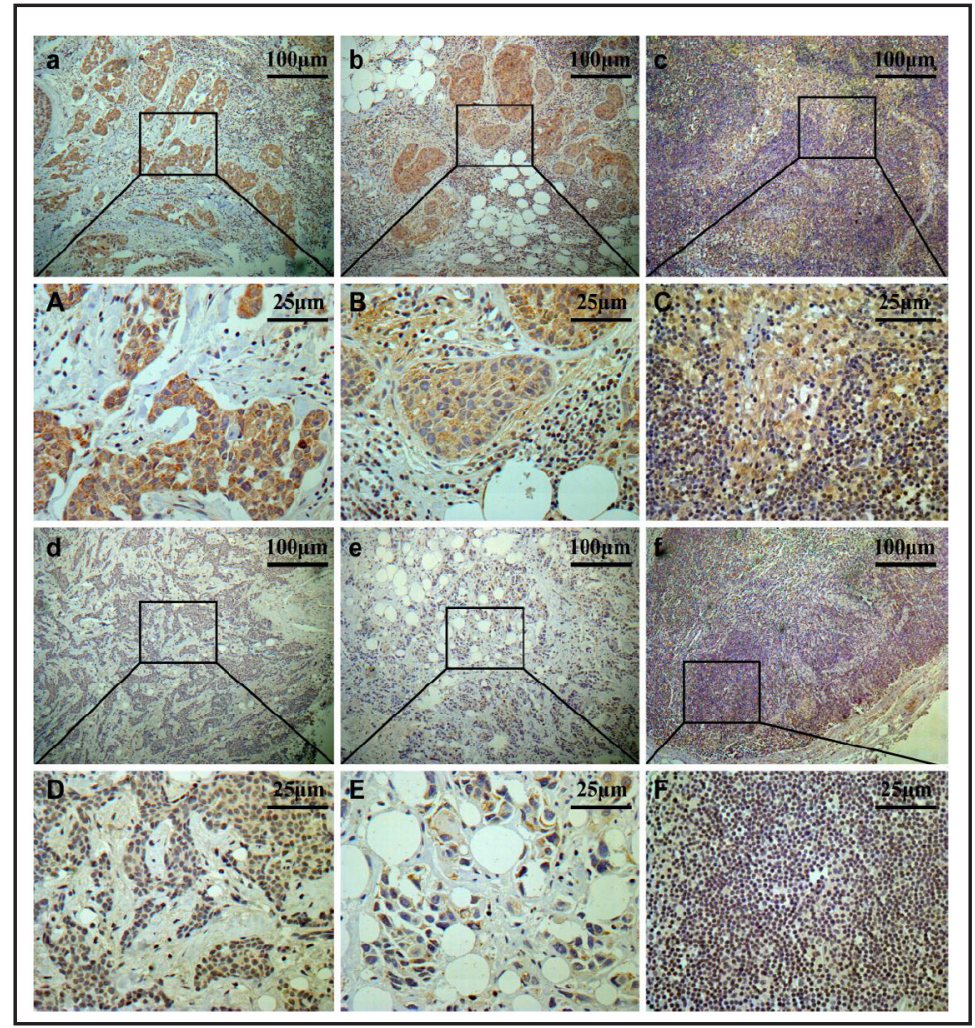


pression level were $83.3 \%$ and $35.8 \%$, respectively. And also we found that the expression level of LAPTM4B was high in the metastatic axillary lymph nodes $(19 / 20$, $95.0 \%$ ) compared with the negative axillary lymph nodes $(2 / 20$, $10.0 \%)$, and the difference was statistically significant $(P<0.001)$.

We conducted univariate and multivariate logistic regression analyses with covariate adjustment of clinicopathological variables for axillary lymph node metastasis. TNM stage was excluded according to clinical advice. The presence of lymph node metastasis was significantly associated with the tumor size, histological grade, molecular subtype, and LAPTM4B expression level (High vs. Low, $P<0.001$ ). All the variables in univariate analysis was included and retained in the multivariate model in a stepwise manner (backward, likelihood ratio) to control for confounding. The multivariate analysis results are presented in Table 2. High LAPTM4B expression level was an independent predictor for axillary lymph node metastasis in $\mathrm{BC}$ (OR: 2.428, 95\% CI=1.333-4.425, $P=0.004)$.

Expression of LAPTM4B in human $B C$ cell lines

We analyzed LAPTMP4B expression in four tumor-derived breast cancer cell lines (MCF-7, T47D, MDA-MB-231, and ZR-7530 cells) by Western blotting, these four cell lines exhibited different levels of LAPTM4B expression (Fig. 2A and Fig. 2B). Because of the highest endogenous expression level of LAPTM4B in MCF-7, we selected this cell line for RNAi
Table 1. Association analyses between the expression levels of LAPTM4B and clinicopathologic factors of breast cancer. * $\chi 2$ test. G1, well differentiated; G2, moderately differentiated; G3, poorly differentiated; IDC, invasive ductal carcinoma; ILC, invasive lobular carcinoma; Others include: mucinous or colloid carcinoma, medullary carcinoma, metaplastic carcinoma

\begin{tabular}{|c|c|c|c|c|}
\hline \multirow{2}{*}{ Variables } & \multirow{2}{*}{$\begin{array}{l}\text { Patients } \\
\text { No.291 }\end{array}$} & \multicolumn{3}{|c|}{ LAPTM4B expression } \\
\hline & & Low $(n=72) \%$ & $\operatorname{High}(\mathrm{n}=219) \%$ & $\mathrm{P}^{*}$ \\
\hline \multicolumn{5}{|l|}{ Age, years } \\
\hline$\leq 50$ & 174 & $40(23.0)$ & $134(77.0)$ & 0.398 \\
\hline$>50$ & 117 & $32(27.4)$ & $85(72.6)$ & \\
\hline \multicolumn{5}{|l|}{ Menopausal status } \\
\hline Pre-menopausal & 131 & $29(22.1)$ & $102(77.9)$ & 0.351 \\
\hline Post-menopausal & 160 & $43(26.9)$ & $117(73.1)$ & \\
\hline \multicolumn{5}{|l|}{ Tumor size, cm } \\
\hline$\leq 2$ & 92 & $34(37.0)$ & $58(63.0)$ & 0.003 \\
\hline$>2, \leq 5$ & 168 & $30(17.9)$ & $138(82.1)$ & \\
\hline$>5$ & 31 & $8(25.8)$ & $23(74.2)$ & \\
\hline \multicolumn{5}{|l|}{ Histological type } \\
\hline IDC & 226 & $57(25.2)$ & $169(74.8)$ & 0.803 \\
\hline ILC & 43 & $9(20.9)$ & $34(79.1)$ & \\
\hline Others & 22 & $6(27.3)$ & $16(72.7)$ & \\
\hline \multicolumn{5}{|l|}{ Histological grade } \\
\hline G1 & 34 & $10(29.4)$ & $24(70.6)$ & 0.781 \\
\hline G2 & 188 & $46(24.5)$ & $142(75.5)$ & \\
\hline G3 & 69 & $16(23.2)$ & $53(76.8)$ & \\
\hline \multicolumn{5}{|l|}{ TNM stage } \\
\hline I & 58 & $29(50.0)$ & $29(50.0)$ & $<0.001$ \\
\hline II & 153 & $27(17.6)$ & $126(82.4)$ & \\
\hline III & 80 & $16(20.0)$ & $64(80.0)$ & \\
\hline \multicolumn{5}{|c|}{ Axillary lymph node metastasis } \\
\hline No & 123 & $44(35.8)$ & $79(64.2)$ & $<0.001$ \\
\hline Yes & 168 & $28(16.7)$ & $140(83.3)$ & \\
\hline \multicolumn{5}{|l|}{ Molecular subtype } \\
\hline Luminal A & 65 & $23(35.4)$ & $42(64.6)$ & 0.139 \\
\hline Luminal B & 123 & $28(22.8)$ & $95(77.2)$ & \\
\hline Her-2+ & 44 & $10(22.7)$ & $34(77.3)$ & \\
\hline TNBC & 59 & $11(18.6)$ & $48(81.4)$ & \\
\hline \multicolumn{5}{|l|}{ P53 status } \\
\hline Negative & 207 & $49(23.7)$ & $158(76.3)$ & 0.506 \\
\hline Positive & 84 & $23(27.4)$ & $61(72.6)$ & \\
\hline \multicolumn{5}{|l|}{ Recurrence } \\
\hline No & 235 & $66(28.1)$ & $169(71.9)$ & 0.007 \\
\hline Yes & 56 & $6(10.7)$ & $50(89.3)$ & \\
\hline
\end{tabular}
analysis, and selected T47D cells for LAPTM4B up-regulation experiments.

Knockdown of LAPTM4B inhibited breast cancer cell proliferation, migration and invasion in vitro

Using MTT assay, we found that LAPTM4B knockdown reduced cell proliferation in the MCF-7 cell line compared with the control and negative control groups. These differences 
Table 2. Risk factors for patients with axillary lymph node metastasis (univariate analysis and multivariate logistic regression analysis adjusted by age). *logistic regression test. B and S.E.: the parameter estimator of association coefficient and its standard error. Abbreviations: OR, odds ratio; C.I., confidence interval. Bold items highlight $\mathrm{P}<0.05$

\begin{tabular}{|c|c|c|c|c|c|c|c|c|}
\hline Variables & \multicolumn{4}{|c|}{ Univariate analysis } & \multicolumn{4}{|c|}{ Multivariate analysis } \\
\hline \multicolumn{9}{|l|}{ Age, years } \\
\hline$>50$ & 0.442 & 0.245 & $1.555(0.961-2.516)$ & 0.072 & & & & \\
\hline \multicolumn{9}{|l|}{ Menopausal status } \\
\hline Post-menopausal & 0.434 & 0.239 & $1.544(0.966-2.468)$ & 0.069 & 0.499 & 0.269 & $1.647(0.972-2.789)$ & 0.064 \\
\hline \multicolumn{9}{|l|}{ Tumor size $(\mathrm{cm})$} \\
\hline$\leq 2$ & \multicolumn{3}{|c|}{1.00 (reference) } & \multicolumn{5}{|c|}{1.00 (reference) } \\
\hline$>2, \leq 5$ & 0.773 & 0.264 & $2.167(1.292-3.634)$ & 0.003 & 0.588 & 0.291 & $1.800(1.018-3.185)$ & 0.043 \\
\hline ILC & -0.478 & 0.334 & $0.620(0.322-1.194)$ & 0.153 & & & & \\
\hline Others & -0.614 & 0.449 & $0.541(0.224-1.306)$ & 0.172 & & & & \\
\hline \multicolumn{9}{|l|}{ Histological grade } \\
\hline G1 & \multicolumn{3}{|c|}{1.00 (reference) } & \multicolumn{5}{|c|}{1.00 (reference) } \\
\hline G2 & 0.672 & 0.382 & $1.957(0.926-4.140)$ & 0.079 & 0.380 & 0.410 & $1.462(0.648-3.300)$ & 0.360 \\
\hline G3 & 1.598 & 0.450 & $4.941(2.045-11.939)$ & $<0.001$ & 1.533 & 0.481 & $4.631(1.804-11.886)$ & 0.001 \\
\hline \multicolumn{9}{|l|}{ Molecular subtype } \\
\hline Luminal A & \multicolumn{3}{|c|}{1.00 (reference) } & \multicolumn{5}{|c|}{1.00 (reference) } \\
\hline Luminal B & 0.882 & 0.315 & $2.416(1.304-4.476)$ & 0.005 & 0.868 & 0.345 & $2.383(1.211-4.689)$ & 0.012 \\
\hline \multicolumn{9}{|c|}{ LAPTM4B Expression } \\
\hline Low & \multicolumn{3}{|c|}{1.00 (reference) } & \multicolumn{5}{|c|}{1.00 (reference) } \\
\hline High & 1.024 & 0.280 & $2.785(1.610-4.818)$ & $<0.001$ & 0.887 & 0.306 & $2.428(1.333-4.425)$ & 0.004 \\
\hline
\end{tabular}

were statistically significant (Fig. 2C). An in vitro wound-healing assay was performed to investigate the effect of LAPTM4B on cellular migration. The results demonstrated that knockdown of LAPTM4B caused an apparent suppression of cell migration in MCF-7 cell lines (Fig. 3A), and the statistical analysis indicated that significant difference was found between the LAPTM4B-silenced group and the mock group (24h: $P=0.001$; $48 \mathrm{~h}: P=0.004)$. Then, the result of Matrigel invasion assays also demonstrated that ablation of endogenous LAPTM4B markedly reduced the invasive capacity of MCF-7 cell lines (Fig. 4A). The number of MCF-7 cells in mock group, which migrated across both the Matrigel and the insert, was significantly higher than those of LAPTM4B-silenced cells $(59.00 \pm 3.00$ vs. $34.33 \pm 2.52$, P $<0.001$ ).

Over-expression of LAPTM4B promoted the proliferation, migration, and invasion of BC cells in vitro

We constructed a T47D-LAPTM4B cell line to determine whether over-expression of LAPTM4B can enhance the invasive capacity of BC cells, which over-expressed of LAPTM4B was demonstrated by Western blot (Fig. 5). MTT assay demonstrated that over-expression of LAPTM4B promotes the proliferation of the T47D cell (Fig. 2D). Wound-healing assay demonstrated that the over-expression of LAPTM4B enhanced T47D cell migration at the 
Fig. 2. The expression level of LAPM4B in four breast cells and the role of LAPTM4B in the proliferation ability of breast cells in vitro. $(\mathrm{A}+\mathrm{B})$ Representative western blot analysis of LAPTM4B expression in four breast cancer cell lines. Protein samples were analysed using SDS-PAGE followed by immunoblotting with antibody against LAPTM4B. The levels of $\beta$-actin were used as an internal control; (C+D) Cell proliferation in MCF-7 cells and T47D cells treated with LAPTM4B-shRNA

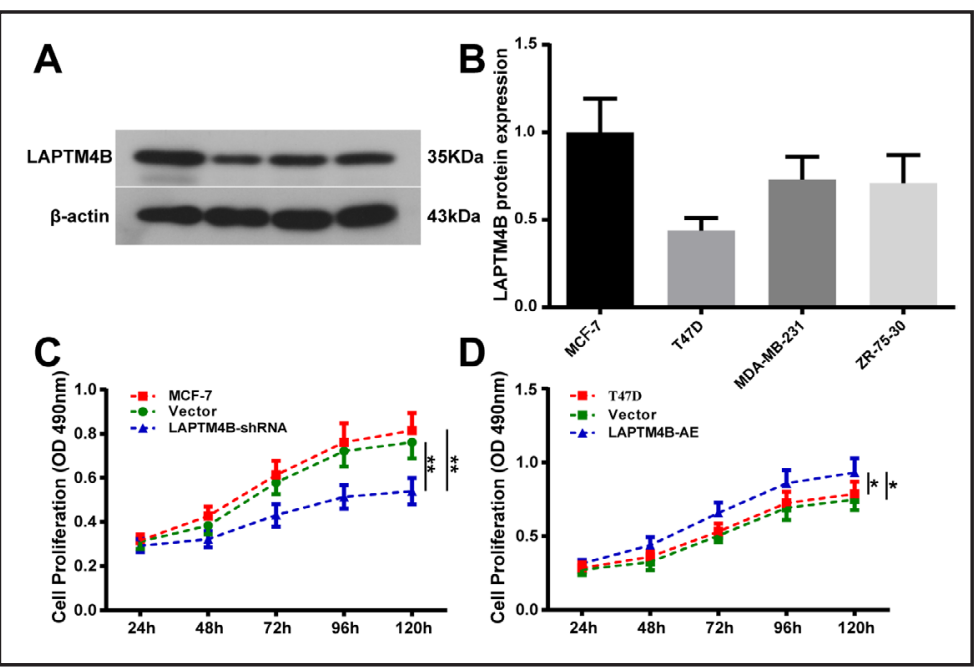
and LAPTM4B-AE. Each point indicates the mean of spectrometric absorbance \pm SD of three independent experiments. ${ }^{*} \mathrm{P}<0.05,{ }^{* *} \mathrm{P}<0.005$.

Fig. 3. The role of LAPTM4B in the migration ability of breast cells in vitro determined by a wound-healing assay. (A) Inhibition of LAPTM4B dramatically reduced the migration capacity of MCF-7 cells; (B) Over-expression of LAPTM4B remarkably promoted the migration capacity of T47D cells.

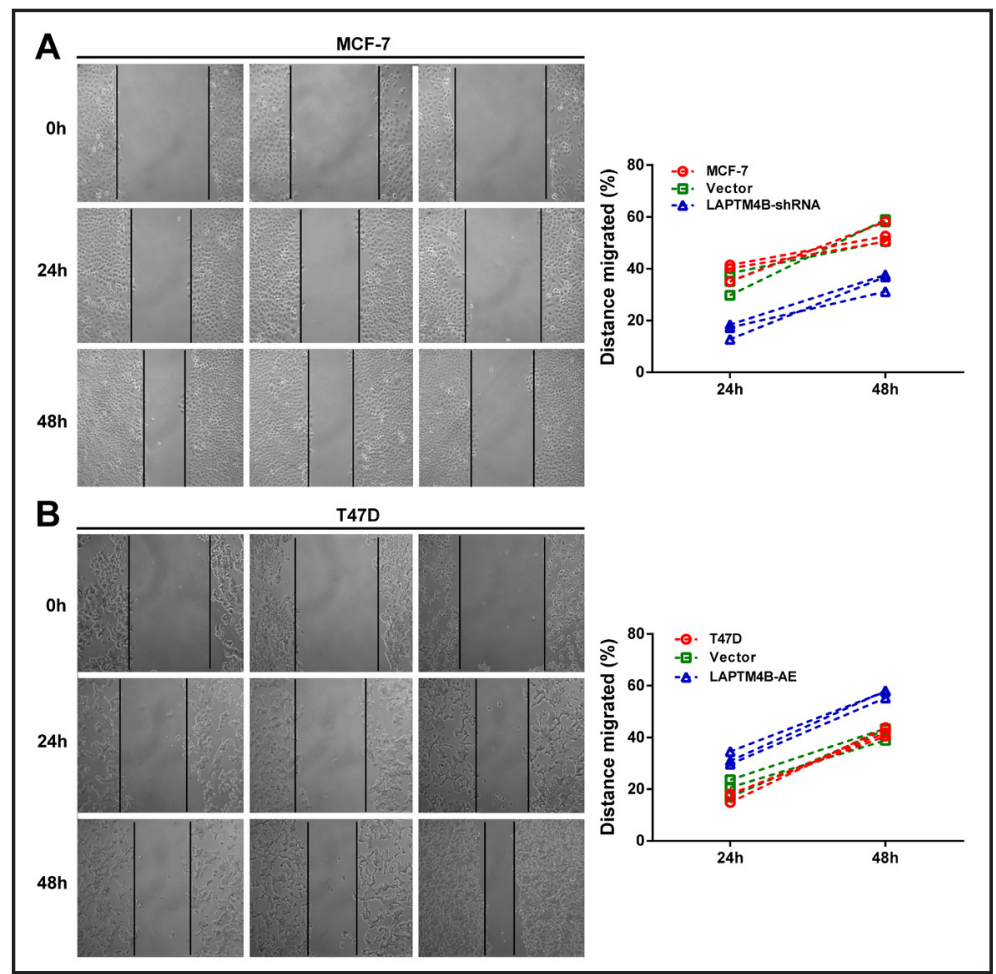

edge of exposed regions (Fig. 3B). Meanwhile, transwell assay also showed that T47DLAPTM4B cells had significantly increased invasive capacity compared with our mock T47D cells (Fig. 4B).

\section{LAPTM4B regulated epithelial-mesenchymal transition in breast cancer cells}

Knockdown of LAPTM4B in MCF-7 cells resulted in up-regulation of the epithelial marker E-cadherin protein levels, and down-regulation of the mesenchymal marker $\mathrm{N}$-cadherin and vimentin protein levels compared with the mock MCF-7 cells (Fig. 5). On the contrary, the expression of E-cadherin was downregulated after LAPTM4B over-expression in T47D cells, whereas the level of N-cadherin and vimentin was upregulated (Fig. 5). 
Fig. 4. The role of LAPTM4B in the invasion ability of breast cells in vitro determined by a Transwell migration assay. (A) Inhibition of LAPTM4B significantly reduced the cells' invasion capacity of MCF-7 cells; (B) overexpression of LAPTM4B dramatically promoted the cells' invasion capacity of T47D cells.

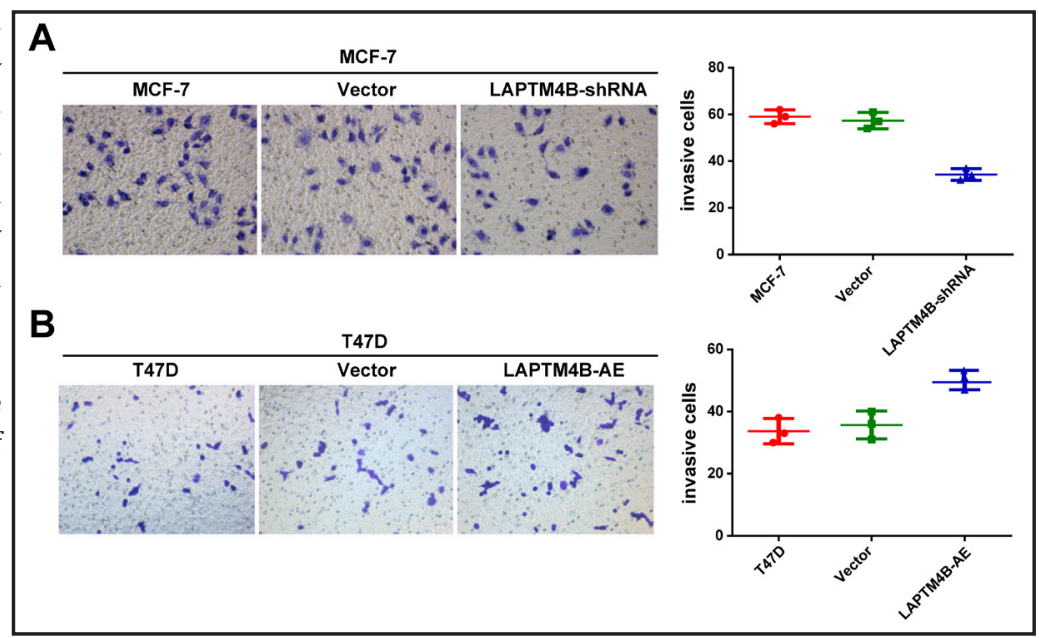

Fig. 5. The effects of LAPTM4B up- or down-regulation on protein levels of E-cadherin, $\mathrm{N}$-cadherin, and vimentin in MCF-7 and T47D cells. The tests were performed in non-transfected control group (Mock: MCF-7 cells and T47D cells), empty vector transfected control group (Vector), LAPTM4B-shRNA group and LAPTM4B-AE group. $\beta$-actin level was used as a loading control. LAPTM4B induced breast cancer cells EMT.

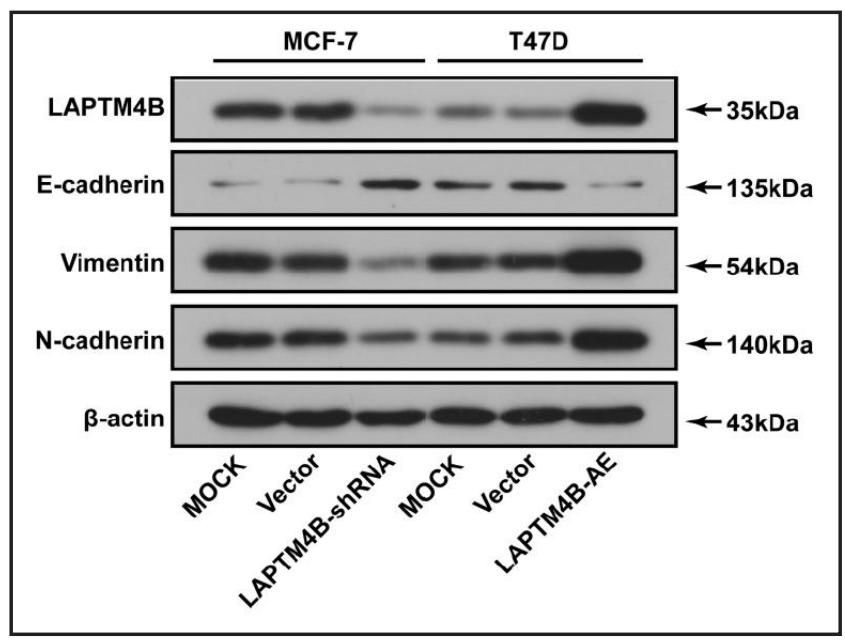

\section{Discussion}

Metastasis is a multi-step process involving angiogenesis, extracellular matrix remodelling, increased motility, and EMT [18]. LAPTM4B is upregulated in many human cancers and exhibits critical roles in the tumorigenesis, promotion, metastasis and prognosis of many human malignancies [19-21]. Our previous research identified that LAPTM4B contributes to poor prognosis of BC. In current study, we investigated in detail the effect of high LAPTM4B expression on axillary lymph node metastasis, we also investigated the effect of the expression level of LAPTM4B on the proliferation, migration and invasion of BC cell lines.

Lymph node metastasis [22] is recognized as the most important marker for the poor prognosis in BC. Therefore, early prediction of lymph node metastasis suggests important clinical significance for improving the prognosis of $\mathrm{BC}$ through setting up the reasonable surgery and drug treatment. Lymph/vascular invasion [23], tumor size [23, 24], histologic type [24] and histological grade [23] were independent predictors of axillary lymph node metastasis in BC. Our results indicated that high LAPTM4B expression was also an independent factor for axillary lymph node metastasis in BC (OR: 2.428, 95\% CI: 1.333-4.425, $P=0.004)$. Considering the metastatic potential, we obtained results consistent with previous findings. Yin et al. [25] reported that high LAPTM4B expression is a new predictor for the intraperitoneal metastasis and lymph node metastasis of epithelial ovarian carcinoma. Our results demonstrated that the sensitivity of high LAPTM4B expression in predicting the axillary lymph node metastasis of BC was $83.3 \%(140 / 168)$, but its specificity was only 


\section{Cellular Physiology Cell Physiol Biochem 2017;41:1072-1082

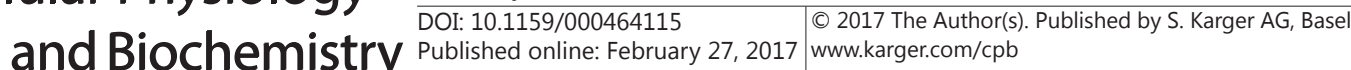 \\ Xiao et al.: LAPTM4B Promotes Breast Cancer Aggressiveness}

$35.8 \%(44 / 123)$. This result suggested that the use of LAPTM4B expression to predict axillary lymph node metastasis should be associated with information obtained from imaging and other gene analyses with high specificity.

Previous studies showed that LAPTM4B gene may play a crucial role in cancer pathogenesis and progression, possibly functioning as a proto-oncogene. Our study yielded similar results. We performed LAPTM4B knockdown and over-expression experiments in vitro. The results demonstrated that the suppression of LAPTM4B significantly inhibited the proliferation, migration and invasion of the MCF-7 cells. By contrast, the over-expression in T47D cells promoted cell proliferation, migration, and invasion. These results are consistent with previous findings in other tumors $[16,20,21,26]$. However, whether the aggressive role of LAPTM4B can be achieved by regulating EMT has not been demonstrated. EMT described as a biological process involved in wound healing, cancer progression, metastasis and drug resistance of various human cancers, including $B C[3,5,27,28]$. The loss of E-cadherin expression and the increase in mesenchymal proteins including Vimentin are considered hallmarks of EMT $[3,29]$. Our study showed that LAPTM4B knockdown can reverse EMT by increasing E-cadherin expression and decreasing N-cadherin and vimentin. These result indicated that LAPTM4B induces EMT, promotes cell migration and invasion, and plays a significant role in $\mathrm{BC}$ metastasis.

LAPTM4B may perform a dual-functional role in the disease progression of malignant tumor cells and multidrug resistance [30,31]. Li et al. found that LAPTM4B stimulates the multidrug resistance of cancer cells by promoting drug efflux and elicits anti-apoptosis effects by activating PI3K/AKT signaling [32]. Lately, a study indicated that LAPTM4B is also essential for homeostasis and lysosomal acidification, and induces the resistance of tumor cells to lysosome-mediated cell death triggered by environmental and genotoxic stresses [33]. In BC, tumor cells become sensitised to anthracycline resistance through the small interfering RNA-mediated knockdown of LAPTM4B. Therefore, further studies should verify whether LAPTM4B can as a therapeutic target in BC treatment and improve patients' outcomes.

In conclusion, our study provided evidence that the LAPTM4B expression is associated with axillary lymph node metastasis in patients with BC and LAPTM4B knockdown is related to the proliferation, migration and invasion of $\mathrm{BC}$ cells, potentially by inhibiting EMT. Therefore, the control of proliferation and metastasis through inhibition of the LAPTM4B gene may be applied as a novel therapeutic approach against BC.

\section{Acknowledgments}

The authors thank all the people who had participated in this study. This work was supported by the National Natural Science Foundation of China (No. 81502266), Natural Science Foundation of Heilongjiang Province (No. QC2015114), HaiYan Foundation of the Affiliated Tumor Hospital of Harbin Medical University (No. JJQN2014-04), and Post Doctoral Foundation of Heilongjiang Province (No. LBH-Z14171).

\section{Disclosure Statement}

We declare that there are no conflicts of interest.

\section{References}

1 Siegel RL, Miller KD, Jemal A: Cancer statistics, 2015. CA Cancer J Clin 2015;65:5-29.

$\checkmark 2$ Torre LA, Bray F, Siegel RL, Ferlay J, Lortet-Tieulent J, Jemal A: Global cancer statistics, 2012. CA Cancer J Clin 2015;65:87-108. 


\section{Cellular Physiology Cell Physiol Biochem 2017;41:1072-1082

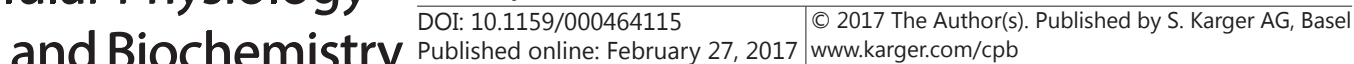

-3 Thiery JP, Acloque H, Huang RY, Nieto MA: Epithelial-mesenchymal transitions in development and disease. Cell 2009;139:871-890.

4 Nieto MA: The ins and outs of the epithelial to mesenchymal transition in health and disease. Annu Rev Cell Dev Biol 2011;27:347-376.

5 Felipe Lima J, Nofech-Mozes S, Bayani J, Bartlett JM: EMT in Breast Carcinoma-A Review. J Clin Med 2016;5:65.

6 Ying X, Sun Y, He P: Bone Morphogenetic Protein-7 Inhibits EMT-Associated Genes in Breast Cancer. Cell Physiol Biochem 2015;37:1271-1278.

7 Liu XR, Zhou RL, Zhang QY, Zhang Y, Jin YY, Lin M, Rui JA, Ye DX: Structure analysis and expressions of a novel tetratransmembrane protein, lysosoma-associated protein transmembrane 4 beta associated with hepatocellular carcinoma. World J Gastroenterol 2004;10:1555-1559.

8 Shao GZ, Zhou RL, Zhang QY, Zhang Y, Liu JJ, Rui JA, Wei X, Ye DX: Molecular cloning and characterization of LAPTM4B, a novel gene upregulated in hepatocellular carcinoma. Oncogene 2003;22:5060-5069.

-9 Zhou L, He XD, Cui QC, Zhou WX, Qu Q Zhou RL, Rui JA, Yu JC: Expression of LAPTM4B-35: a novel marker of progression, invasiveness and poor prognosis of extrahepatic cholangiocarcinoma. Cancer Lett 2008;264:209-217.

10 Zhou L, He XD, Yu JC, Zhou RL, Shan Y, Rui JA: Overexpression of LAPTM4B-35 attenuates epirubucininduced apoptosis of gallbladder carcinoma GBC-SD cells. Surgery 2011;150:25-31.

11 Meng F, Luo C, Hu Y, Yin M, Lin M, Lou G, Zhou R: Overexpression of LAPTM4B-35 in cervical carcinoma: a clinicopathologic study. Int J Gynecol Pathol 2010;29:587-593.

12 Meng FL, Yin MZ, Song HT, Yang H, Lou G, Zhou RL: LAPTM4B-35 overexpression is an independent prognostic marker in endometrial carcinoma. Int J Gynecol Cancer 2010;20:745-750.

13 Yin M, Lou C, Zhang W, Meng F, Zhang H, Ning X, Zhou R, Dong X, Lou G: LAPTM4B overexpression is a novel independent prognostic marker for metastatic ovarian tumors. Int J Gynecol Cancer 2012;22:54-62.

- 14 Xiao M, Jia S, Wang H, Wang J, Huang Y, Li Z: Overexpression of LAPTM4B: an independent prognostic marker in breast cancer. J Cancer Res Clin Oncol 2013;139:661-667.

15 Jatoi I: Management of the axilla in primary breast cancer. Surg Clin North Am 1999;79:1061-1073.

16 Yang H, Xiong F, Wei X, Yang Y, McNutt MA, Zhou R: Overexpression of LAPTM4B-35 promotes growth and metastasis of hepatocellular carcinoma in vitro and in vivo. Cancer Lett 2010;294:236-244.

17 Vergarajauregui S, Martina JA, Puertollano R: LAPTMs regulate lysosomal function and interact with mucolipin 1: new clues for understanding mucolipidosis type IV. J Cell Sci 2011;124:459-468.

18 Hur K, Toiyama Y, Takahashi M, Balaguer F, Nagasaka T, Koike J, Hemmi H, Koi M, Boland CR, Goel A: MicroRNA-200c modulates epithelial-to-mesenchymal transition (EMT) in human colorectal cancer metastasis. Gut 2013;62:1315-1326.

19 Li Y, Zhang Q, Tian R, Wang Q, Zhao JJ, Iglehart JD, Wang ZC, Richardson AL: Lysosomal transmembrane protein LAPTM4B promotes autophagy and tolerance to metabolic stress in cancer cells. Cancer Res 2011;71:7481-7489.

20 Meng F, Chen X, Song H, Lou G: LAPTM4B down regulation inhibits the proliferation, invasion and angiogenesis of HeLa cells in vitro. Cell Physiol Biochem 2015;37:890-900.

-21 Meng F, Chen X, Song H, Lou G, Fu S: Lentivirus-mediated RNA Interference Targeting LAPTM4B Inhibits Human Ovarian Cancer Cell Invasion In Vitro. Chem Biol Drug Des 2016;87:121-130.

-22 Sorlie T, Perou CM, Tibshirani R, Aas T, Geisler S, Johnsen H, Hastie T, Eisen MB, van de Rijn M, Jeffrey SS, Thorsen T, Quist H, Matese JC, Brown PO, Botstein D, Lonning PE, Borresen-Dale AL: Gene expression patterns of breast carcinomas distinguish tumor subclasses with clinical implications. Proc Natl Acad Sci USA 2001;98:10869-10874.

23 Silverstein MJ, Skinner KA, Lomis TJ: Predicting axillary nodal positivity in 2282 patients with breast carcinoma. World J Surg 2001;25:767-772.

24 Nouh MA, Ismail H, El-Din NH, El-Bolkainy MN: Lymph node metastasis in breast carcinoma: clinicopathological correlations in 3747 patients. J Egypt Natl Canc Inst 2004;16:50-56.

25 Yin M, Xu Y, Lou G, Hou Y, Meng F, Zhang H, Li C, Zhou R: LAPTM4B overexpression is a novel predictor of epithelial ovarian carcinoma metastasis. Int J Cancer 2011;129:629-635.

-26 Liu X, Xiong F, Wei X, Yang H, Zhou R: LAPTM4B-35, a novel tetratransmembrane protein and its PPRP motif play critical roles in proliferation and metastatic potential of hepatocellular carcinoma cells. Cancer Sci 2009;100:2335-2340. 


\section{Cellular Physiology Cell Physiol Biochem 2017;41:1072-1082 \begin{tabular}{ll|l} 
DOI: 10.1159/000464115 & 2017 The Author(s). Published by S. Karger AG, Basel \\
and Biochemistry & Published online: February 27, 2017 & www.karger.com/cpb \\
\cline { 2 - 3 }
\end{tabular} \\ Xiao et al.: LAPTM4B Promotes Breast Cancer Aggressiveness}

-27 Acloque H, Adams MS, Fishwick K, Bronner-Fraser M, Nieto MA: Epithelial-mesenchymal transitions: the importance of changing cell state in development and disease. J Clin Invest 2009;119:1438-1449.

28 Imani S, Hosseinifard H, Cheng J, Wei C, Fu J: Prognostic Value of EMT-inducing Transcription Factors (EMTTFs) in Metastatic Breast Cancer: A Systematic Review and Meta-analysis. Sci Rep 2016;6:28587.

29 Mimeault M, Batra SK: Interplay of distinct growth factors during epithelial mesenchymal transition of cancer progenitor cells and molecular targeting as novel cancer therapies. Ann Oncol 2007;18:1605-1619.

-30 Yin M, Li C, Li X, Lou G, Miao B, Liu X, Meng F, Zhang H, Chen X, Sun M, Ling Q, Zhou R: Over-expression of LAPTM4B is associated with poor prognosis and chemotherapy resistance in stages III and IV epithelial ovarian cancer. J Surg Oncol 2011;104:29-36.

-31 Kasper G, Vogel A, Klaman I, Grone J, Petersen I, Weber B, Castanos-Velez E, Staub E, Mennerich D: The human LAPTM4b transcript is upregulated in various types of solid tumours and seems to play a dual functional role during tumour progression. Cancer Lett 2005;224:93-103.

-32 Li L, Wei XH, Pan YP, Li HC, Yang H, He QH, Pang Y, Shan Y, Xiong FX, Shao GZ, Zhou RL: LAPTM4B: a novel cancer-associated gene motivates multidrug resistance through efflux and activating PI3K/AKT signaling. Oncogene 2010;29:5785-5795.

33 Zhang S, Huang WC, Li P, Guo H, Poh SB, Brady SW, Xiong Y, Tseng LM, Li SH, Ding Z, Sahin AA, Esteva FJ, Hortobagyi GN, Yu D: Combating trastuzumab resistance by targeting SRC, a common node downstream of multiple resistance pathways. Nat Med 2011;17:461-469. 